\title{
A neuro-fuzzy approach to fast ferry vertical motion modelling
}

\author{
M. Santos*, R. López, J.M de la Cruz \\ Dpto. Arquitectura de Computadores y Automática. Facultad de CC. Físicas, Universidad Complutense de Madrid, 28040-Madrid, Spain
}

Received 15 April 2005; received in revised form 21 June 2005; accepted 31 August 2005

Available online 10 October 2005

\begin{abstract}
In order to stabilise the motion of a high speed craft, and so to improve the comfort of the passengers and the crew while maintaining the speed, control-oriented model are needed. For this purpose, neuro-fuzzy systems have been used to obtain general models of the nonlinear behaviour of a fast ferry. The sources of the available knowledge are the physical laws of the vertical dynamics of the craft, and some experimental and simulated data of the ship performance. Two non-linear models focused on the vertical motion of the craft, both heave and pitch, are proposed: an academic one and a predictive one. The modelling task is complex and the results are original as the problem has not been previously solved in a general way neither by applying artificial intelligence techniques. The models have been proved satisfactory with regular and also irregular waves, and they have been used for ship control purposes.
\end{abstract}

(C) 2005 Elsevier Ltd. All rights reserved.

Keywords: Neuro-fuzzy modelling; Marine systems; Ship control

\section{Introduction}

The research deals with a fast ferry called "Silvia Ana". Currently, the ferry works in La Plata and in the Baltic Sea. The craft has an aluminium-made deep $\mathrm{V}$ hull, and the following characteristics: $119 \mathrm{~m}$ length, $14.696 \mathrm{~m}$ beam, $2.405 \mathrm{~m}$ draught, 475 tons dead weight, 1250 passengers, 250 cars (Anonymous, 1996, 1998).

The main goal of dealing with fast marine systems is to stabilise the motion of the craft for some purposes such as improve the comfort of the passengers and the safety, maintaining the speed. The main impact on the behaviour in this aspect is caused by the vertical acceleration, both heave and pitch motions. The vertical acceleration originates the seasickness, a most important concern, which can be measured by the motion sickness incidence (MSI) (O'Hanlon and McCawley, 1974). In order to control this system and so to improve the quality of the travelling by reducing the vertical acceleration, control-oriented models are needed to develop and evaluate the controllers.

\footnotetext{
*Corresponding author. Tel.: + 34913944374 ; fax: + 34913944687.

E-mail address: msantos@dacya.ucm.es (M. Santos).
}

On the other hand, ship motion is a complicated issue to model. The description of high speed crafts behaviour is complex because the model of the system changes with the ship speed and the sea state in a non-linear way. Some of its parameters are coupled, and others are variables. In addition, the uncertainty that comes from the sea waves encourages dealing with wide broad models, which by means of artificial intelligence techniques can incorporate knowledge about the system.

The motivation for using neuro-fuzzy techniques is due to the uncertainty that comes from the waves and the sea state, and the ability that fuzzy logic provides to deal with the complexity and strongly non-linear nature of the system itself (Babuska and Verbruggen, 1996). Moreover, data and knowledge are available to be incorporated to the system by these intelligent methods.

In this paper two models are proposed. An academic model which is oriented to dealing with ideal waves, that provides the amplitude and phase of the vertical motion (both heave and pitch), and also the pitch moment of the ship; the second one predicts the value of those signals based on the values of the height of the waves at previous moments. The models are obtained by fuzzy inference systems (FIS) which are implemented by applying adaptive 
neuro-fuzzy techniques to the available data. The experimental and simulated data have been collected and provided by CEHIPAR (Cehipar, 1998), a specialised towing tank, working with a small replica of the ship.

Both of them allow a better understanding of the system, and have been used to design fuzzy controllers in order to stabilise the motion of the craft (Esteban et al., 2000; López and Santos, 2002; López et al., 2002). These controllers based on the fuzzy model have been applied in real time experiments with a replica of the ship with good results (Santos et al., 2005).

As far as we know, there have been no previous fuzzy models of these marine systems either general ones. Other models that have been obtained for this ferry consist of a set of different models - linear in some cases - for each application point (wavelength, speed, etc.) under particular conditions (Aranda et al., 2000, 2002; de la Cruz et al., 1998; Andres et al., 2000). The models proposed in this paper can be applied in a wider range of sailing conditions.

The paper is organised as follows. Section 2 presents the general equations for ship motion. Section 3 shows a description of the ferry behaviour based on simulated data, and deals with the design of the neuro-fuzzy model for ideal waves. Some results show its performance. In Section 4 , the development of the predictive and real waves oriented model is shown with some results. Section 5 summarises the conclusions.

\section{Ship dynamic}

Understanding the dynamic of the system in a qualitative way is essential in order to develop a fuzzy model. The main source of knowledge is always a deep study of the physics of the problem. The key issues of the ships motion are considered in Lloyd (1998), Lewis (1989) and Fossen (1994).

The ship is not only under the influence of the waves, wind, ocean currents, etc., but also its own inertia, the added mass, the hydrodynamic damping, and the stiffness forces. The system of six general linearised equations that describes the physical motion of the craft for small amplitude motions in regular waves can be written (Lloyd, 1998)

$$
\begin{aligned}
& \sum_{j=1}^{6}\left(A_{i j} \frac{\mathrm{d}^{2} x_{j}}{\mathrm{~d} t^{2}}+b_{i j} \frac{\mathrm{d} x j}{\mathrm{~d} t}+c_{i j} x_{j}\right)=F \omega_{i 0} \sin \left(\omega_{\mathrm{e}} t+\varphi_{i}\right), \\
& \quad i=1, \ldots, 6
\end{aligned}
$$

where each one of the three terms on the left-hand side refers to the inertia, the damping and the stiffness forces, respectively. The ship has linear accelerations, $\ddot{x}_{1}, \ddot{x}_{2}$, and $\ddot{x}_{3} \mathrm{~m} / \mathrm{s}^{2}$, and angular accelerations, $\ddot{x}_{4}, \ddot{x}_{5}$, and $\ddot{x}_{6} \mathrm{rad} / \mathrm{s}^{2}$.

The excitation amplitude, $F_{\omega i o}$, and the phase, $\varphi_{i}$, are given by

$$
F \omega_{i 0}=\delta_{0} \sqrt{\left(c_{i}-a_{i} \omega_{\mathrm{e}}^{2}\right)^{2}+\left(b_{i} \omega_{\mathrm{e}}\right)^{2}}(\mathrm{kN}),
$$

$\tan \varphi_{i}=\frac{b_{i} \omega_{\mathrm{e}}}{c_{i}-a_{i} \omega_{\mathrm{e}}^{2}}$,

where $\delta_{0}$ is the wave amplitude.

The encounter frequency, $\omega_{\mathrm{e}}$, is defined as the frequency at which the ship and a train of regular waves meet. It is a function of the frequency of the waves, $\omega_{0}$, the speed of the craft, $U$, and the heading angle, $\mu$, angle relative to the direction of propagation of a train of regular waves. The formula in deep water is given by (Lloyd, 1998)

$\omega_{\mathrm{e}}=\omega_{0}-\frac{\omega_{0}^{2}}{g} U \cos \mu \quad(\mathrm{rad} / \mathrm{s})$.

The wave modal frequency, $\omega_{0}$, can be obtained by Pierson-Moskowitz spectrum formula, where $H_{1 / 3}$ is the observed significant height of the waves

$\omega_{0}=0.4 \sqrt{\frac{g}{H_{1 / 3}}}=\frac{1.2526}{\sqrt{H_{1 / 3}}} \quad(\mathrm{rad} / \mathrm{s})$.

In addition, simulated data about the ship motion in ideal regular waves have been provided by CEHIPAR and processed by using the computer program PRECAL (based on finite elements). In this way, using some estimated values of the frequency of the waves, the modal frequency may also be estimated by the expression

$\omega_{0}=1.1103 / \sqrt{H_{1 / 3}} \quad(\mathrm{rad} / \mathrm{s})$,

which is quite similar but provides slightly lower values than (3). From now on, this modal frequency as defined in (4) will be used to characterise the sea state number (SSN), according to the World Meteorological Organisation (WMO) (see Table 1).

The experimental data corresponding to frequencies around each modal frequency are grouped and related to one specific SSN. The behaviour of the system for a fixed SSN can be associated with sinusoidal signals with that model frequency.

Fig. 1 shows the encounter frequency $\omega_{\mathrm{e}}(\mathrm{rad} / \mathrm{s})$ vs. the modal frequency of the waves $\omega_{0}(\mathrm{rad} / \mathrm{s})$ for different ship speed. These data have been obtained by substituting the

\begin{tabular}{|c|c|c|c|c|c|c|}
\hline \multirow[t]{3}{*}{ SSN } & \multicolumn{6}{|c|}{ Waves } \\
\hline & \multicolumn{3}{|c|}{$H_{1 / 3}(\mathrm{~m})$} & \multicolumn{3}{|c|}{$\omega_{0}(\mathrm{rad} / \mathrm{s})$} \\
\hline & Min & Max & Med & Min & Max & Med \\
\hline 0 & - & - & - & - & - & - \\
\hline 1 & 0.00 & 0.10 & 0.05 & 3.51 & - & $4.9^{7}$ \\
\hline 2 & 0.10 & 0.50 & 0.30 & 1.57 & 3.51 & 2.0 \\
\hline 3 & 0.50 & 1.25 & 0.88 & 0.99 & 1.57 & 1.1 \\
\hline 4 & 1.25 & 2.50 & 1.88 & 0.70 & 0.99 & 0.8 \\
\hline 5 & 2.50 & 4.00 & 3.25 & 0.56 & 0.70 & 0.6 \\
\hline 6 & 4.00 & 6.00 & 5.00 & 0.45 & 0.56 & 0.5 \\
\hline 7 & 6.00 & 9.00 & 7.50 & 0.37 & 0.45 & 0.4 \\
\hline 8 & 9.00 & 14.00 & 11.50 & 0.30 & 0.37 & 0.3 \\
\hline 9 & 14.00 & - & 14.00 & - & 0.30 & 0.3 \\
\hline
\end{tabular}

Table 1

Sea states 


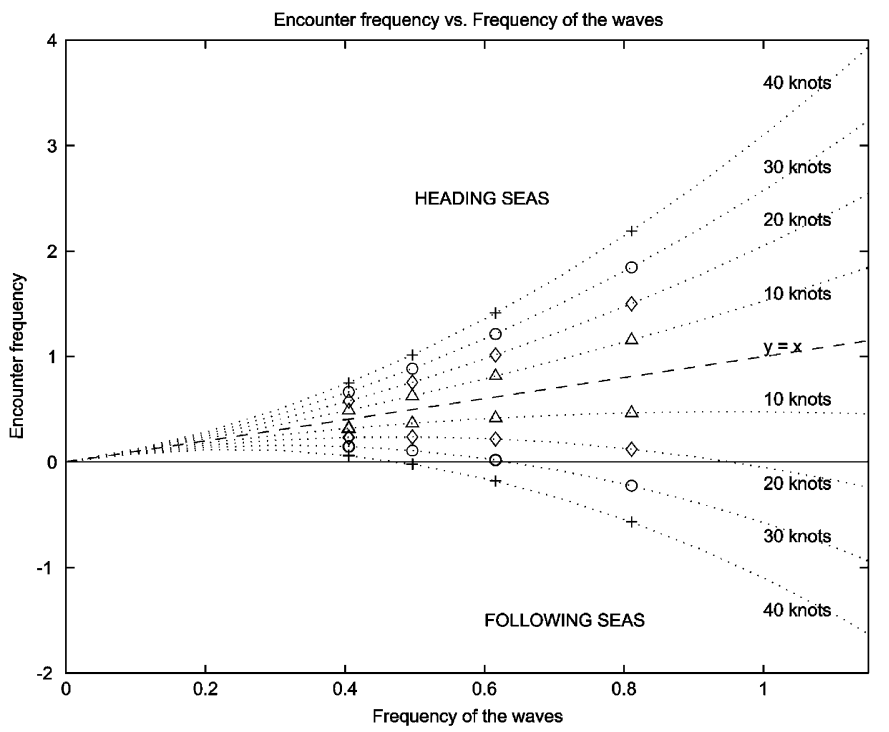

Fig. 1. Encounter frequency $\omega_{\mathrm{e}}$ vs. wave frequency $\omega_{0}$.

data provided by CEHIPAR for the replica into (4) for $\omega_{0}$ and applying (2) to calculate $\omega_{\mathrm{e}}$. As it can be seen, it reaches negative values for speed larger than 20 knots in following seas and high modal frequencies (SSN 5, 4 and 3).

The relationship between $\omega_{\mathrm{e}}$ and $U \cos \mu$ (the component of the velocity of the ship in the direction of wave propagation) is plotted in Fig. 2, for different wave frequencies. In this figure, negative values of $U \cos \mu$ mean heading sea. As it shows, for a fixed SSN and in head waves, increasing the ship speed causes a linear increment in the encounter frequency. On the other hand, for fixed SSN and following seas, the encounter frequency falls linearly if speed increases. In this case, although the positive and large values of $U \cos \mu$, the encounter frequency can reach negative values.

Going back to (1), the coefficients (local inertia, damping and stiffness) are not constant, and depend on the wave frequency (or wavelength), the ship speed and the hull shape.

Being $m$ the total mass in tonnes and $I$ the mass moments of inertia of the ship, the acceleration coefficients $A_{i j}$ consist of the inertia mass and the added mass $\left(A_{i j}=m_{i j}+a_{i j}, i=j=1,2,3\right)$, or the moment of inertia and the added mass $\left(A_{i j}=I_{i j}+a_{i j}, i=j=4,5,6\right)$, which depends also on the heading angle. It is needed to remark that this system performs with large inertial forces (Fig. 3).

If the real mass of the ship is represented as $m_{55}$ or $I_{55}$, Fig. 3 shows that, at $\omega_{\mathrm{e}} \approx 2 \mathrm{rad} / \mathrm{s}$, the added or virtual mass, $a_{55}$, is twice the real mass (2.08 times), and it increases when decreasing $\omega_{\mathrm{e}}$ to $0 \mathrm{rad} / \mathrm{s}$. For example, at 40 knots, the added mass is $4.33,20.34$ and 20.01 times $I_{55}$ when $\omega_{\mathrm{e}}=1.0158,0.5030$ and $-0.5080 \mathrm{rad} / \mathrm{s}$, respectively. That means that the frequency range of interest will be around $1 \mathrm{rad} / \mathrm{s}$, where the largest vertical acceleration is reached, as it will be shown in Section 3.

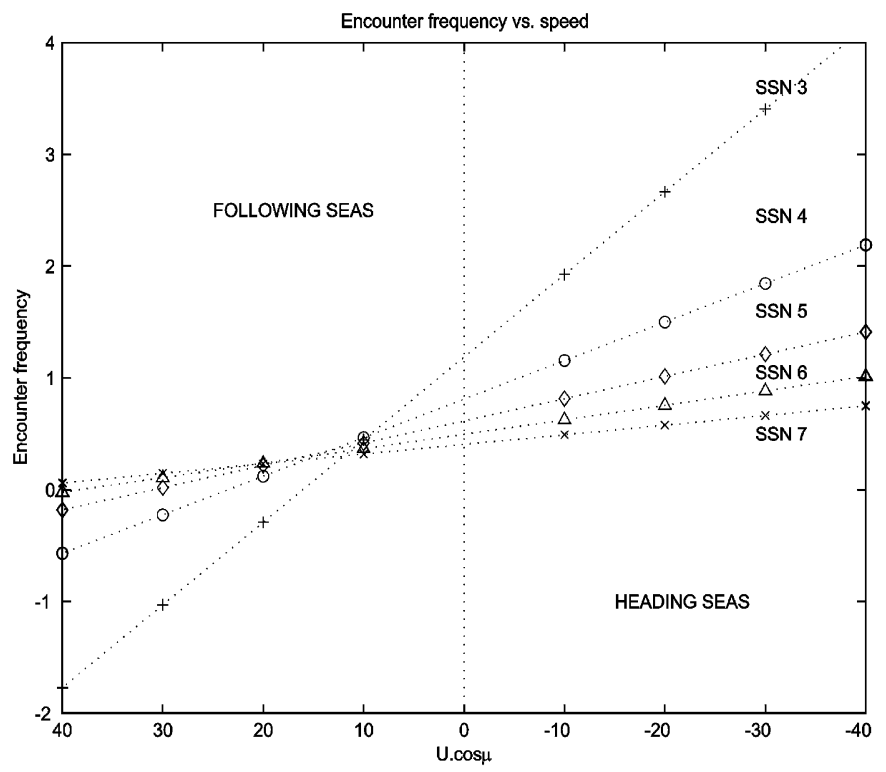

Fig. 2. Encounter frequency $\omega_{\mathrm{e}}$ vs. $U \cos \mu$.

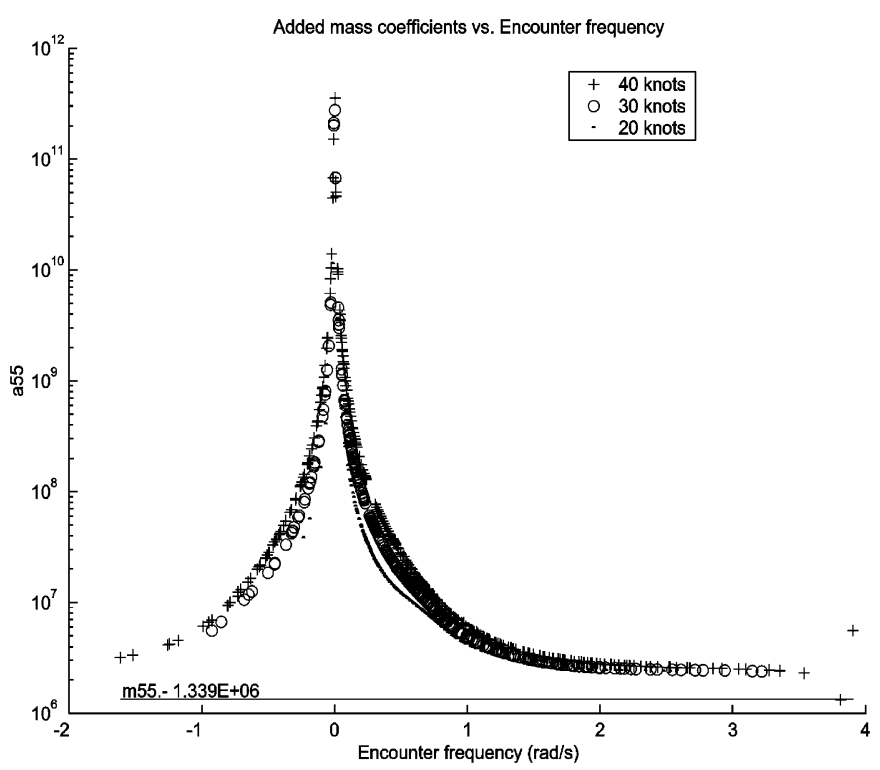

Fig. 3. Added mass coefficients $A_{i j}$ vs. encounter frequency $\omega_{\mathrm{e}}$.

When plotting the behaviour of the damping coefficients, $\mathrm{b}_{i j}$, the maximum is reached at frequency $\omega_{\mathrm{e}} \approx 0-1 \mathrm{rad} / \mathrm{s}$.

Based on experimental data and the port/starboard symmetry of the craft, some of the coefficients have been found to be zero or negligible and other are constant. The motions that remain coupled are pitch and heave. Therefore, the initial study is focused on heave and pitch motions with heading sea.

\section{Neuro-fuzzy modelling}

A qualitative understanding of the behaviour of the ship helps to develop the model and to test it. The more 
interesting observed aspect is the coupling of the ship length and the distance between consecutive waves, as it is reflected in the literature. In fact, if the ship lies on two or more waves, there will be small heave and pitch motions. In the same way, increasing the distance between waves removes the relief of the ship. Then, vertical accelerations become significant. In any case, the forces exerted by the waves originate effects that will depend on the dynamic characteristics of the ship.

Recasting system (1), the equations for heave $(j=3)$ and pitch $(j=5)$ motions are

$\left(m_{33}+a_{33}\right) \ddot{x}_{3}(t)+b_{33} \dot{x}_{3}(t)+c_{33} x_{3}(t)=F_{30} \sin \left(\omega_{\mathrm{e}} t+\varphi_{3}\right)$,

$\left(I_{55}+a_{55}\right) \ddot{x}_{5}(t)+b_{55} \dot{x}_{5}(t)+c_{55} x_{5}(t)=F_{50} \sin \left(\omega_{\mathrm{e}} t+\varphi 5\right)$.

Solving the system with initial conditions $x_{j}=0, \dot{x}_{j}=1$ for different ship speed and different encounter frequencies, it is possible to prove (Ziegler, 1968) that the steady state solutions for the pitch signal are sinusoidal functions

$x_{5}(t)=x_{50} \sin \left(\omega_{\mathrm{e}} t+\varphi_{5}\right)$,

$\ddot{x}_{5}(t)=-x_{50} \omega_{\mathrm{e}}^{2} \sin \left(\omega_{\mathrm{e}} t+\varphi_{5}\right)=-\omega_{\mathrm{e}}^{2} x_{5}(t)$,

where $x_{50}$ is the maximum pitch amplitude and $\varphi_{5}$ is the phase. The same type of solution can be found for heave motion. These equations will be used to obtain the graphics of the ship behaviour when applying the experimental data supplied by Precal $\left(x_{50}, \varphi_{5}, x_{30}, \varphi_{3}\right)$.

To get some insight into the model, pitch excitation, $F_{50}$, and pitch acceleration, $\ddot{x}_{5}$, are also represented as a function of the encounter frequency for different ship speed in order to get approximated models of the behaviour. Simulations with Precal have been accomplished by the towing tank Cehipar for regular waves, at speed of 20,30 and 40 knots, and different sea state codes $\left(\omega_{0}=\{0.3930-1.1470 \mathrm{rad} / \mathrm{s}\}\right)$. Some of the simulation results are shown in Figs. 4-7 (dashed).

\subsection{The neuro-fuzzy approach}

The models that represent these data are obtained by FIS which are generated by applying subtractive clustering.

The purpose of clustering is to identify natural groupings of data from a large data set to produce a concise representation of a system's behaviour. The cluster information can be used to generate a Sugeno-type FIS that best models the data behaviour using a minimum number of rules. Subtractive clustering (Chiu, 1994) is a fast, one-pass algorithm for estimating the number of clusters and clusters centres in a set of data. As in this case the number of clusters is unknown, subtractive clustering is applied.

An FIS is a model that maps inputs characteristics to input membership functions, input membership functions to rules, rules to a set of output characteristics, output characteristics to output membership functions, and the output membership function to a single-valued output or a

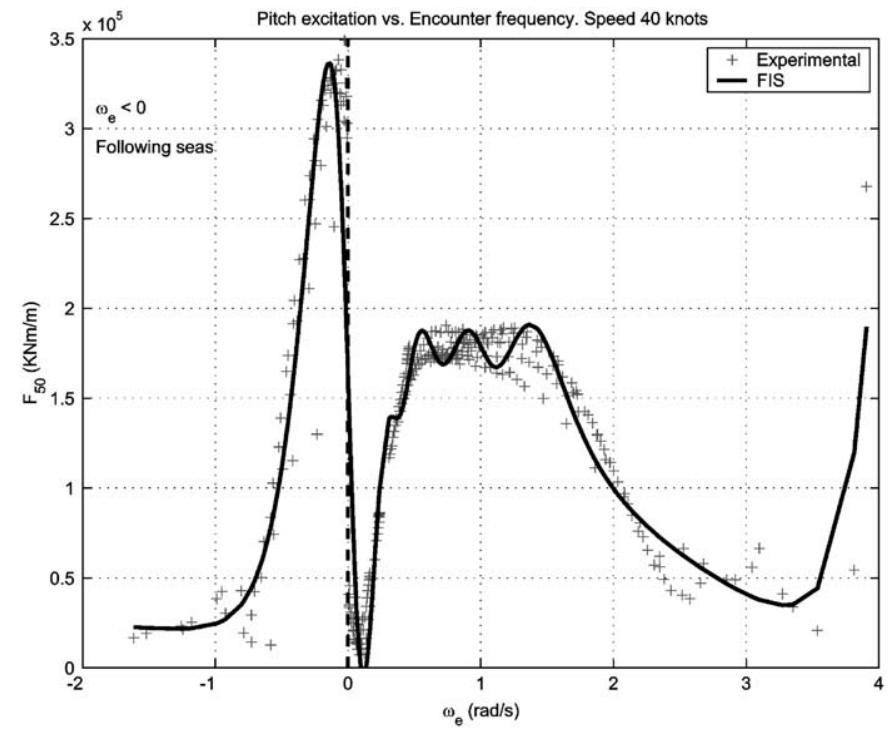

Fig. 4. Pitch excitation vs. encounter frequency.

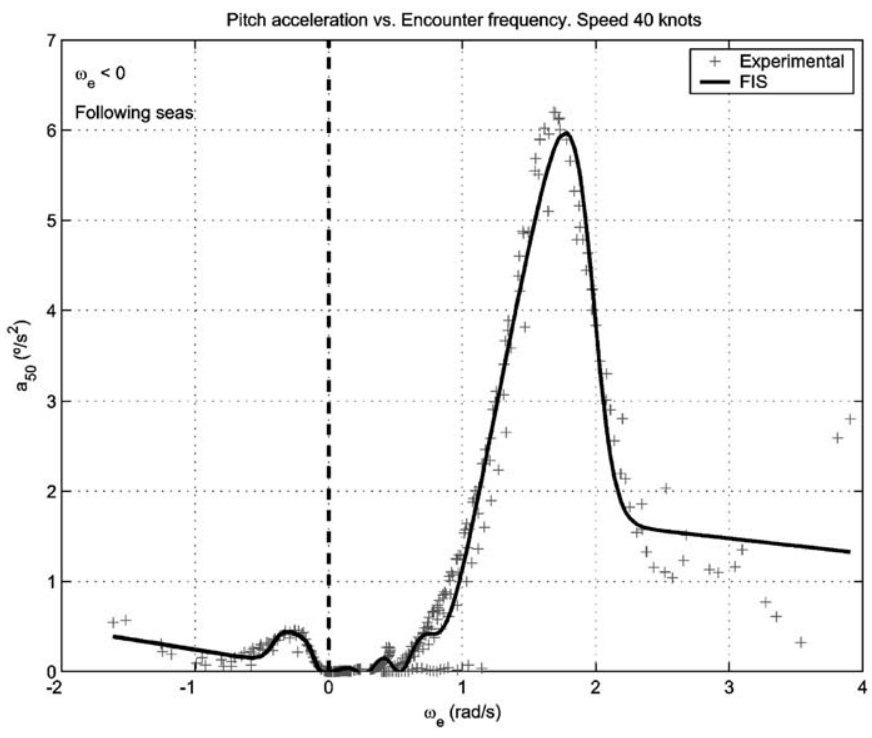

Fig. 5. Pitch acceleration vs. encounter frequency.

decision associated with the output. The parameters associated with a given membership function are chosen in order to account for the variations in the data values. The method that is used to incorporate the characteristics of the problem in the inference system is called neuroadaptive learning. These neuro-adaptive learning techniques provide a method for the fuzzy modelling procedure to learn the information about a data set, in order to compute the membership function parameters that best allow the associated FIS to track the given input/output data. This learning strategy works similarly to that of neural networks.

The computational procedure that accomplishes this membership function parameters adjustment is called ANFIS (Jang, 1993; Brown and Harris, 1994). The 


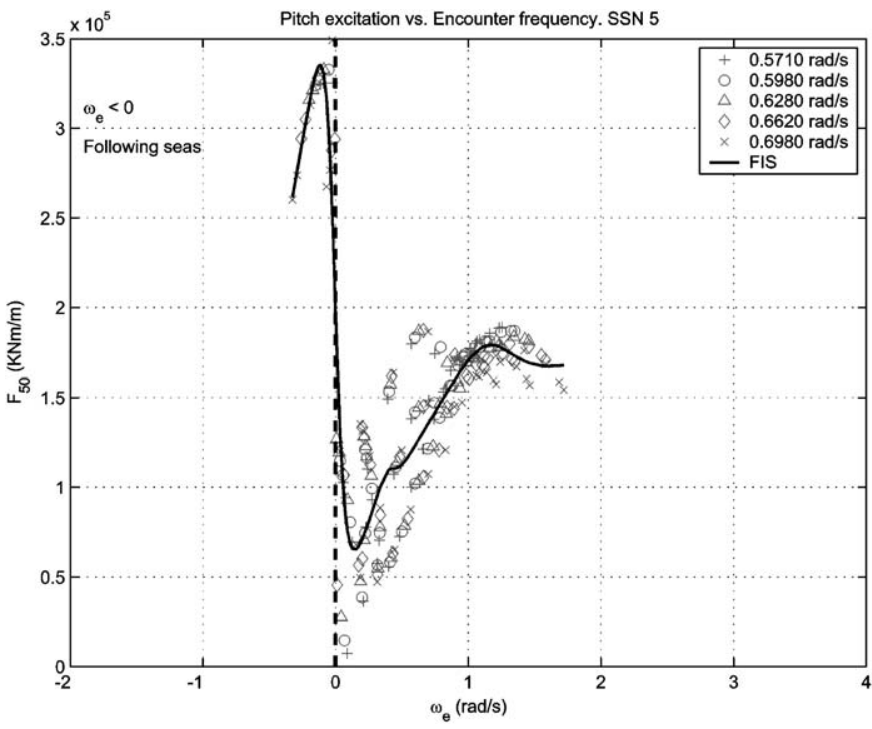

Fig. 6. Pitch excitation vs. encounter frequency (SNN of 5).

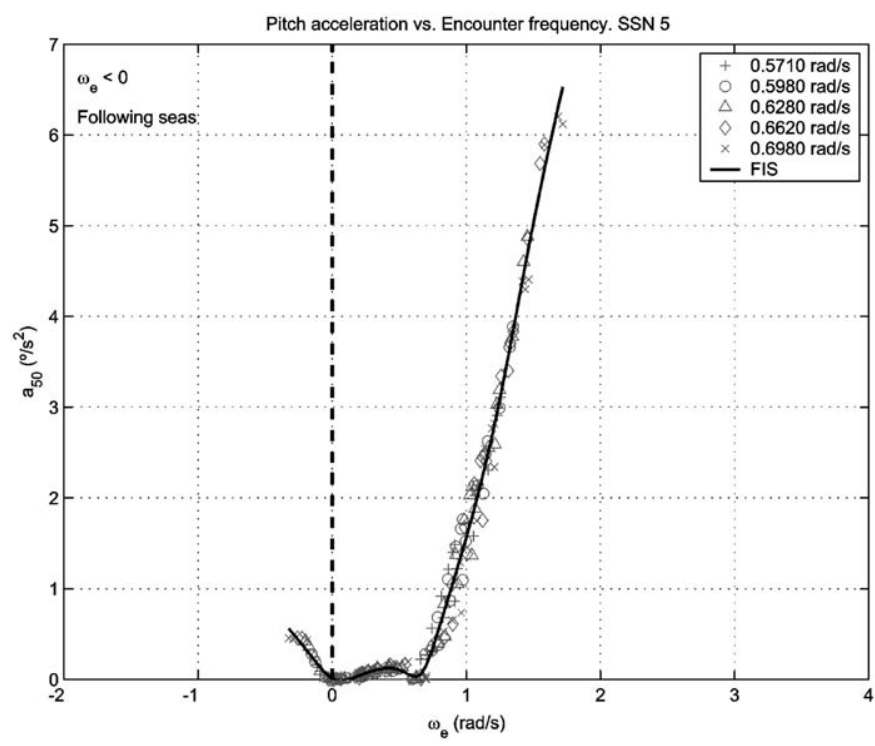

Fig. 7. Pitch acceleration vs. encounter frequency (SNN of 5).

acronym ANFIS derives its name from "adaptive neurofuzzy inference system". Using a given input/output data set, this procedure constructs an FIS whose membership function parameters are tuned using either backpropagation algorithm alone, or in combination with a least squares type of method. This allows the fuzzy systems to learn from the data they are modelling.

Applying subtractive clustering and training the FIS by neuro-adaptive learning, a first approximation of a general fuzzy model is obtained. For instance, Figs. 4 and 5 show the behaviour of the ship (dashed: experimental data, solid: FIS) at 40 knots. Similar representations can be obtained for each one of the sailing conditions.

Analysing all the graphics of the available experiments, it can be concluded that: (i) Excitation signals reach a maximum around $\omega_{\mathrm{e}} \approx$ $1 \mathrm{rad} / \mathrm{s}$ for positive values of the encounter frequency. There is also a peak at $\omega_{\mathrm{e}} \approx 0 \mathrm{rad} / \mathrm{s}$ for following seas. These maximum are left shifting when ship speed increases. The shape is an inverted parabola in both cases, meaning that excitation forces decrease for large frequencies (positive or negative).

(ii) Pitch acceleration is similar but it reaches its maximum at $\omega_{\mathrm{e}} \approx 1.5 \mathrm{rad} / \mathrm{s}$. For negative values of the encounter frequency, the acceleration is almost zero. The expected amplitude range for the acceleration is $0-7^{\circ} / \mathrm{s}^{2}$, which matches with the predictions of the model described in Esteban et al. (2000), and so it has been considered when applying the controllers (Aranda et al., 2002; Santos et al., 2005).

Figs. 6 and 7 show other examples of neuro-fuzzy inferences of the ship behaviour. In this case, the pitch excitation and pitch acceleration are represented as a function of the encounter frequency for a fixed Sea State of 5 (modal frequencies between 0.5710 and $0.6980 \mathrm{rad} / \mathrm{s}$ ).

It is interesting to notice the way acceleration grows at $1 \mathrm{rad} / \mathrm{s}$, although the excitation is larger at other frequencies (see Fig. 4).

Finally, Fig. 8 illustrates the experimental data of the pitch acceleration vs. the encounter frequency and Fig. 9 shows this variable vs. ship speed, for different SNN. For high frequencies, i.e., SSN of 5-7, the experimental data are scant because of the difficulty of the measurements.

\subsection{The general neuro-fuzzy model based on ideal waves}

The model based on ideal waves experiments consists of three FIS (see Fig. 10). The fuzzy model of the ship has three inputs: the sea state (given by the modal frequency of the waves), the ship speed, and the heading angle. Each of

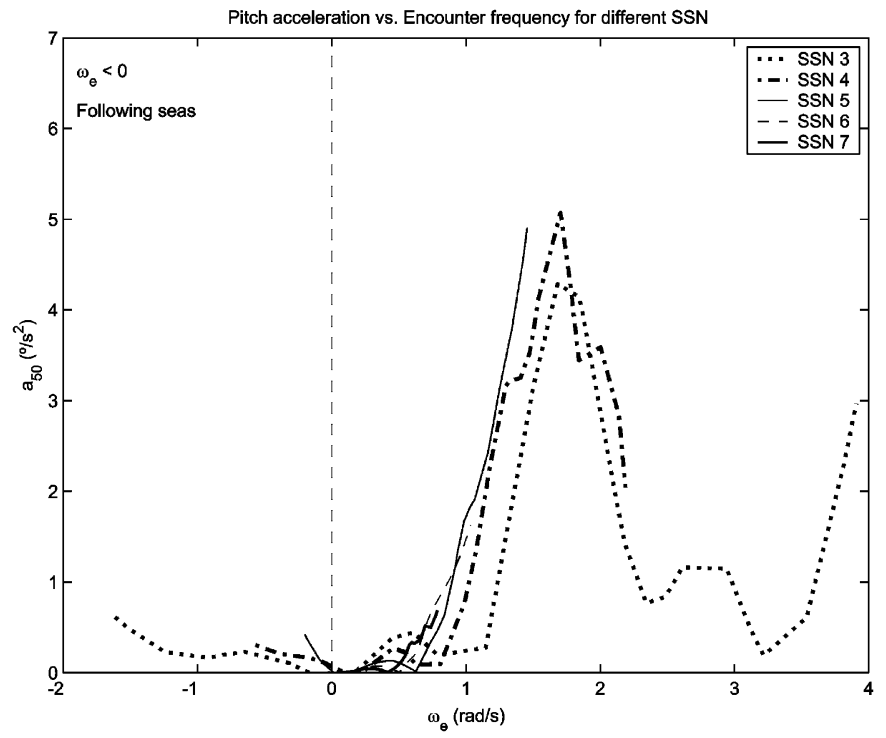

Fig. 8. Pitch acceleration vs. encounter frequency. 


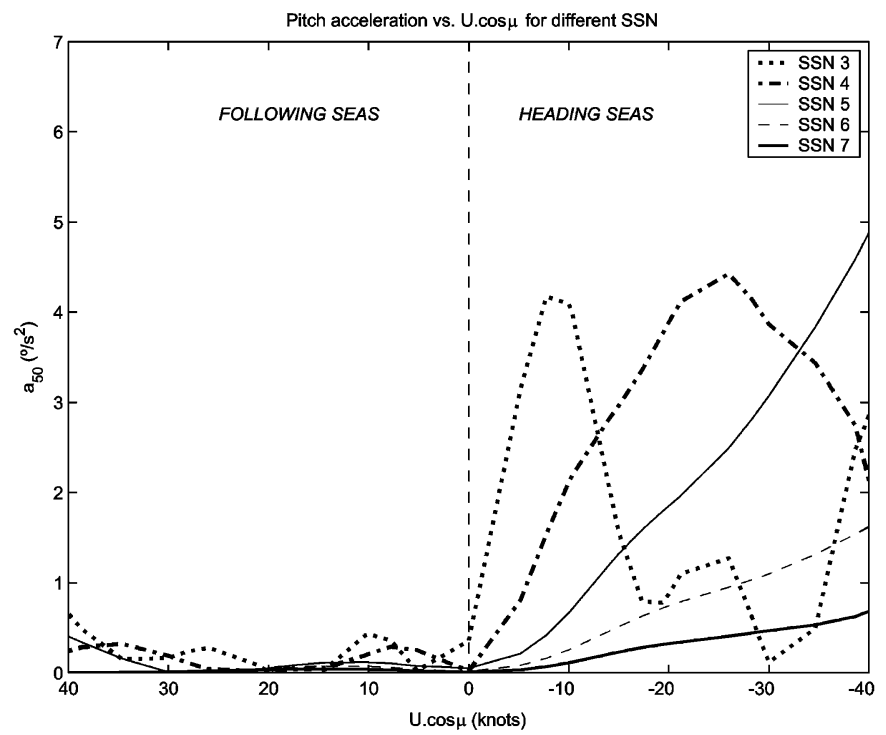

Fig. 9. Pitch acceleration vs. $U \cos \mu$.

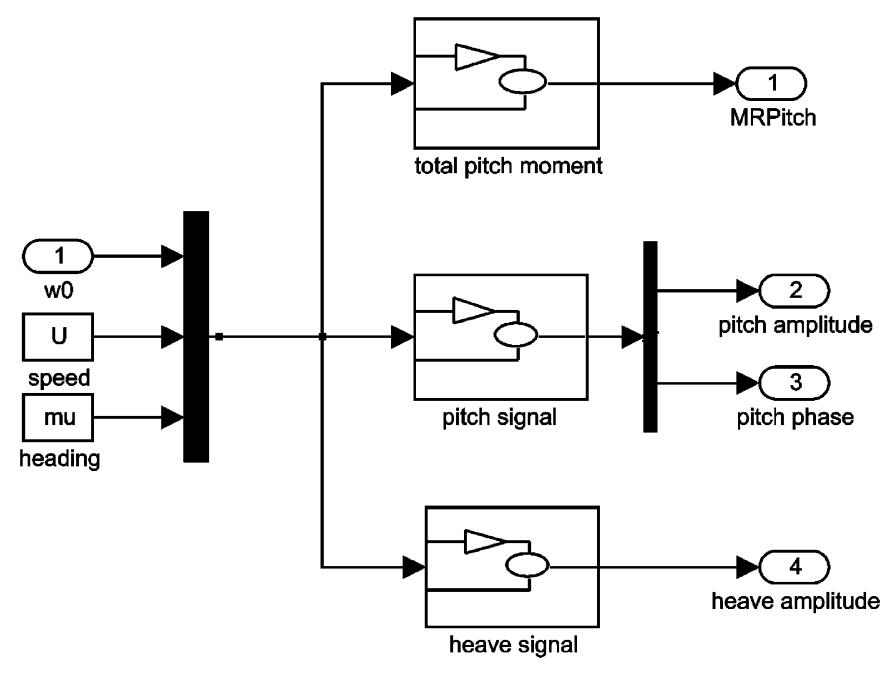

Fig. 10. Fuzzy model of the ship.

them has been represented by five membership functions uniformly distributed in their corresponding universe of discourse (Fig. 11). The output variables are the heave and pitch motions (both, amplitude and phase), and the final pitch moment.

The model emulates the total pitch moment of the ship, i.e., the one caused by the final pitch acceleration, not only by the external excitations. It takes into account that the forces caused by the waves are filtered by the ship and so its moment does not necessarily fit the total moment. For example, at 40 knots for following seas - negative values of $\omega_{\mathrm{e}}$ frequency - the pitch acceleration is almost zero despite the large excitation forces (as it can be seen in Figs. 4 and 5).

Block structure of the pitch and the heave systems are similar. They consist of some functions that estimate the signal amplitude, the phase, and the encounter frequency

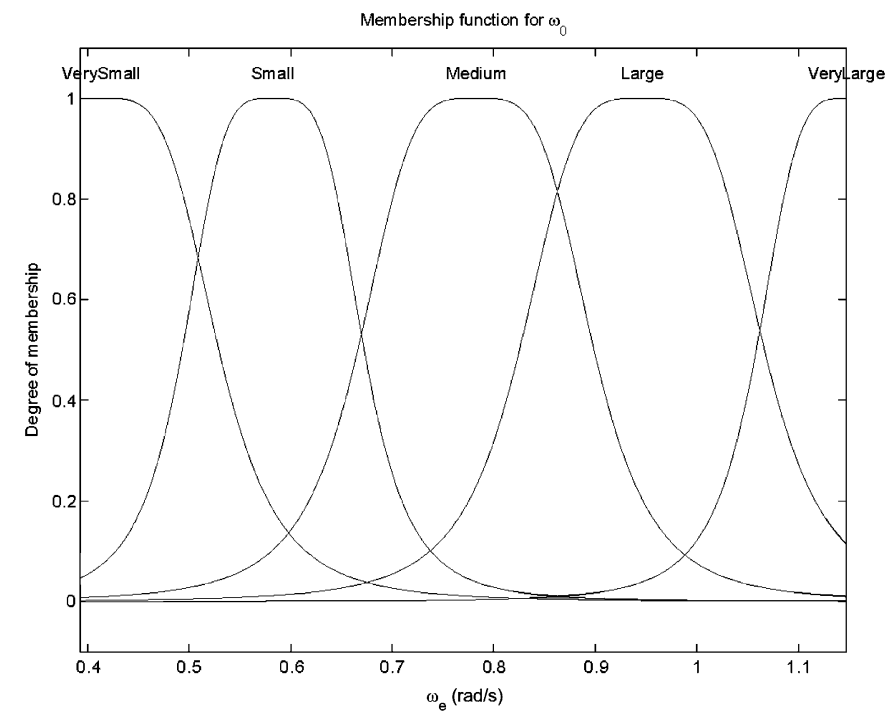

Fig. 11. Membership functions for $\omega_{0}$.

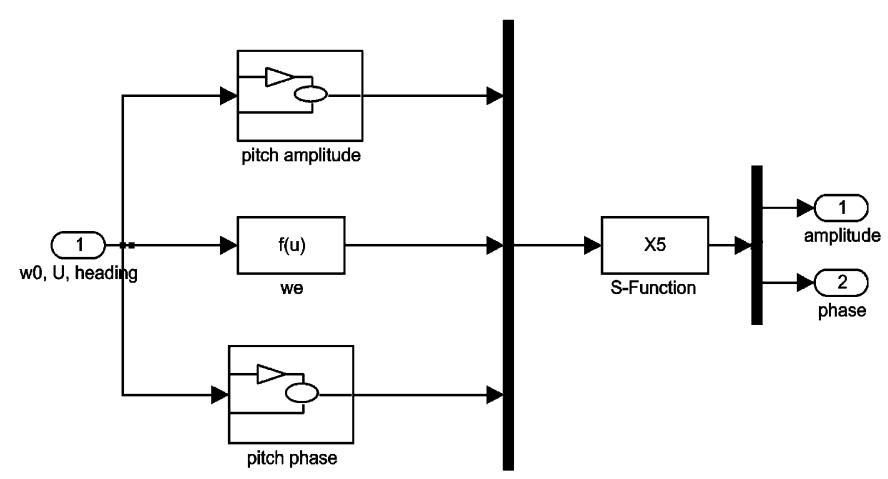

Fig. 12. Block of the pitch signal.

by applying (4). Based on that, the output function calculates the corresponding output signal (heave, $x_{3}$, pitch, $x_{5}$, or even the pitch moment, $M_{5}$ ). See Fig. 12 for pitch signal.

These functions, designed to calculate the pitch and heave amplitude and phase, are implemented by means of a Sugeno-type FIS. The output of these fuzzy systems is a linear function of the inputs that gives a crisp value. These kind of systems are computational efficient as well as they assure the continuity of the output surface (Harris et al., 1993). They have been trained applying ANFIS learning strategies (see Section 3.1).

The neuro-fuzzy model has been tested by introducing simulated regular waves as another input to the model. Fig. 13 shows the results of the model performing at 40 knots, SSN of $5\left(\omega_{0}=3.78 \mathrm{~m}\right)$ and heading seas $\left(\mu=180^{\circ}\right)$. The encounter frequency in this case is $1.255 \mathrm{rad} / \mathrm{s}$ (upper graphic). The behaviour of the fuzzy model is compared to the experimental model obtained by Precal (Esteban et al., 2000) for the same conditions. The comparison shows a good result. 


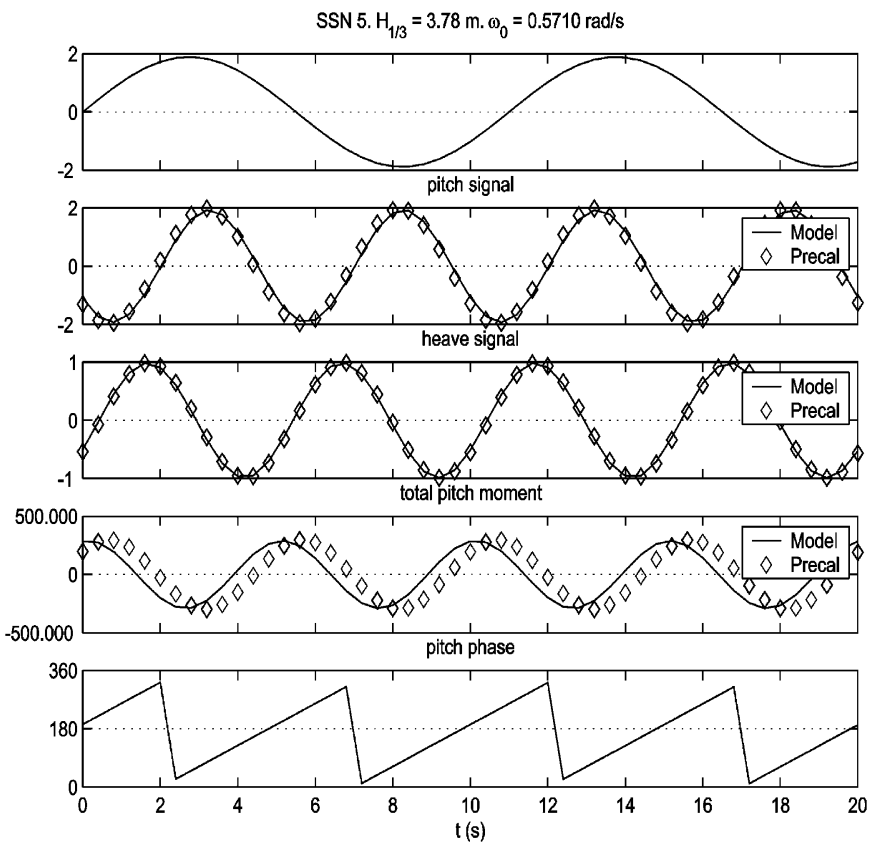

Fig. 13. Comparison between fuzzy model (solid) and the experimental one (diamonds).

The fuzzy model fits very well at SSN of 4-6. For lower and higher sea state the experimental data are more scattered and that makes the fitness more difficult. For SNN lower than 4 there is no problem of stabilisation, and for SNN larger than 6, the ferry could hardly travel. The results are then very encouraging in the range of interest.

It must be taken into account that the neuro-fuzzy model is a general one in the sense that can be apply for any condition, whereas the other models that have been developed consist of a set of different models for specific conditions (Aranda et al., 2000, 2002; de la Cruz et al., 1998; Andres et al., 2000).

Although this model has been proved very useful for designing the control law for the ferry (Santos et al., 2005), when it was tested with irregular waves the results were not completely satisfactory mainly due to the phase, so a new model is proposed.

\section{Predictive model}

In this section, a different approach is considered to obtain a model working with real waves. The adaptive neuro-fuzzy techniques are again used to develop a general model for any sea state or velocity with irregular waves.

\subsection{Data}

In this case, the available data are experimental, not simulated, provided by the CEHIPAR towing tank. They have been obtained by carrying out some experiments with a $1 / 25$ scale replica of the ferry with series of real waves at a constant speed. In each experiment, several variables
Table 2

Experiments

\begin{tabular}{lll}
\hline SSN & $U$ (knots) & Number of measures \\
\hline 4 & 20 & 922 \\
4 & 30 & 527 \\
4 & 40 & 309 \\
5 & 20 & 922 \\
5 & 30 & 527 \\
5 & 40 & 309 \\
6 & 20 & 922 \\
6 & 30 & 527 \\
6 & 40 & 309 \\
\hline
\end{tabular}

(height of the wave, acceleration and position of heave and pitch, etc.) have been measured by sensors at intervals of $0.25 \mathrm{~s}$.

Owing to the limited length of the channel $(152 \mathrm{~m})$, the number of measurements is reduced when increasing the speed of the experiment (see Table 2).

In addition to these experimental data, simulated data from Precal have been used for calculate the values of the added mass (coefficients $a_{i j}$ ).

The shape of the acceleration (heave and pitch) and the moment signals depend on the waves and the speed of the ship. At a constant speed $U$, these signals will be a function of the amplitude values of the waves, taken at time intervals $\Delta t$.

So, the variable $v$ that is wanted to be predicted (such as pitch acceleration) can be expressed as

$v(t)=f\left(h_{\mathrm{w}}(t-n \Delta t), \ldots, h_{\mathrm{w}}(t-(n-1) \Delta t), h_{\mathrm{w}}(t-\Delta t)\right)$.

The problem consists of finding out $\Delta t$ and $n$ that make possible to predict enough accurately the value of the variable.

Initially, tests were made with $n=4,6$ and 8 using $\Delta t=0.25 \mathrm{~s}$. The results were not completely satisfactory. So, the following criteria were established to choose them:

(i) The interval $[0, n \times \Delta t]$ might cover at least the complete cycle of the wave.

(ii) In addition, $n$ must be the smallest possible.

That leads to

$\Delta t=\frac{2 \pi}{n \times \omega_{0}}$.

Inspecting in Table 2 the range of experiments (SSN 4-6), the smallest frequency to be considered occurs at SSN 6 , with waves of 4 and $6 \mathrm{~m}$ high and modal frequency around $\omega_{0}=0.45 \mathrm{rad} / \mathrm{s}$. Therefore, applying (8)

$\Delta t=\frac{13.96}{n}$.

After considering different values of $n$, it was established that at least $n$ should be 9 , then $\Delta t=1.55 \mathrm{~s}$. As for the experiments $\Delta t_{\mathrm{e}}=0.25 \mathrm{~s}$, finally $\Delta t=1.50 \mathrm{~s}$ and $n=10$. 


\subsection{Neuro-fuzzy predictive model}

An FIS is applied to generate the so-called predictive model. The FIS has $n+3$ input variables (13) - corresponding to the modal frequency, $\omega_{0}$, the speed of the ferry, $U$, the heading angle, $\mu$, and the 10 past measures of the wave height. The output is the pitch acceleration.

The ANFIS algorithm allows replacing the knowledge of the expert with the implicit knowledge contained in the data set (Section 3.1). However, the number of data for training should be several times greater than the number of parameters that is wanted to obtain (at least 5 times). In this case, the model has 200 adjustable parameters and the series of available data for training are 1182 . Once the data have been arranged properly, the FIS is trained with a third of the data. The generalisation is made with the secondthird, reserving the last-third for the model test.

For each experiment, a non-dimensional error is defined as

$\varepsilon_{\text {exp }}=\frac{1}{\# \text { measurement }} \sum_{i=1}^{\text {\#measurement }} \frac{\operatorname{abs}\left(\operatorname{acc}_{\exp }(i)-\operatorname{acc}_{\bmod }(i)\right)}{\operatorname{abs}(\operatorname{acc} \exp (i))}$,

Table 3

Experiments errors

\begin{tabular}{llll}
\hline SSN & $U$ (knots) & $\omega_{\mathrm{e}}(\mathrm{rad} / \mathrm{s})$ & $\varepsilon_{\exp }$ \\
\hline 4 & 20 & 1.5715 & 0.42 \\
4 & 30 & 1.9388 & 0.53 \\
4 & 40 & 2.3061 & 0.63 \\
5 & 20 & 1.0421 & 0.38 \\
5 & 30 & 1.2490 & 0.46 \\
5 & 40 & 1.4560 & 0.58 \\
6 & 20 & 0.7281 & 0.27 \\
6 & 30 & 0.8505 & 0.40 \\
6 & 40 & 0.9730 & 0.49 \\
\hline
\end{tabular}

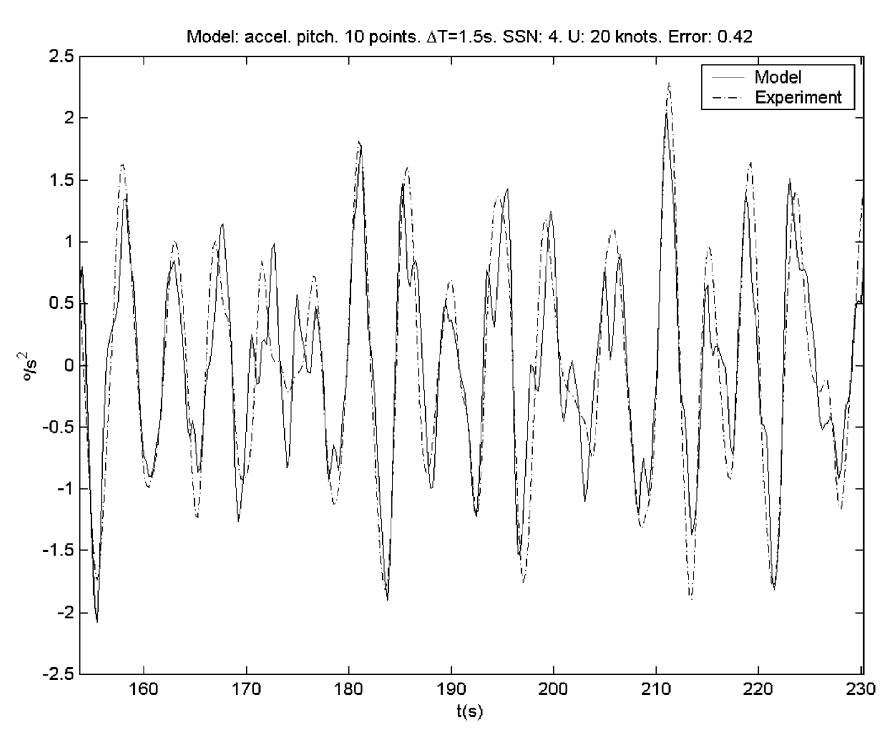

Fig. 14. Results for $\mathrm{SSN}=4 U=20$ knots. where $\operatorname{acc}_{\exp }(i)$ and $\operatorname{acc}_{\text {mod }}(i)$ are the experimental and the modal values of the pitch acceleration, respectively. Table 3 shows the error obtained with the experimental data after training the model.

Finally, Figs. 14-16 show a comparison between the experimental values of the pitch acceleration and the model ones for the three experiments whose encounter frequency is close to the natural frequency of oscillation. That is, $\mathrm{SSN}=4$ with speed of 20 and 30 knots, and SSN $=5$ with speed of 40 knots.

As it can be observed, the model follows the results of the experiments and fits well the real data.

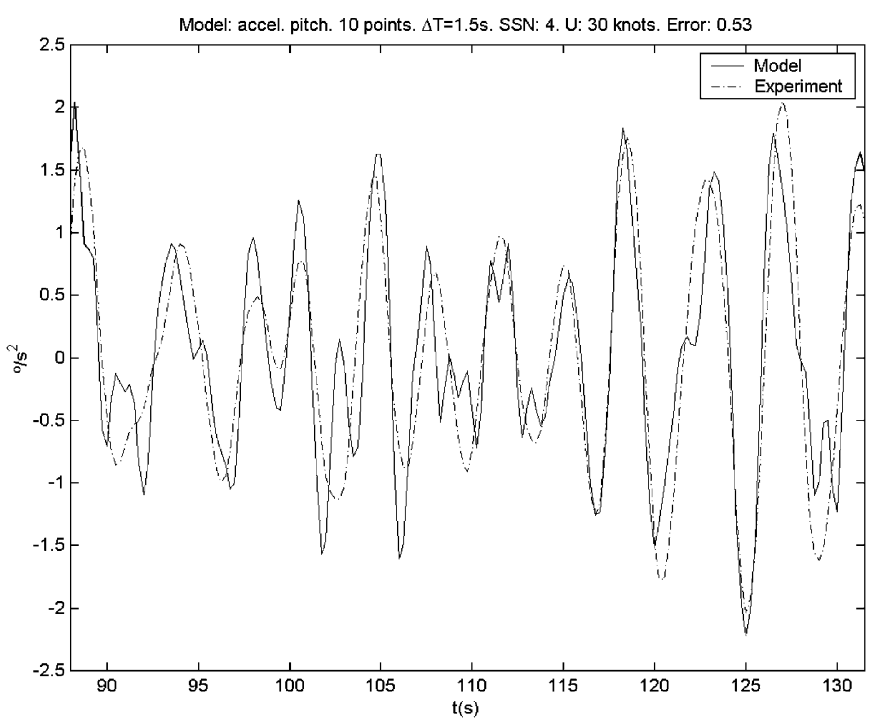

Fig. 15. Results for $\mathrm{SSN}=4 U=30$ knots.

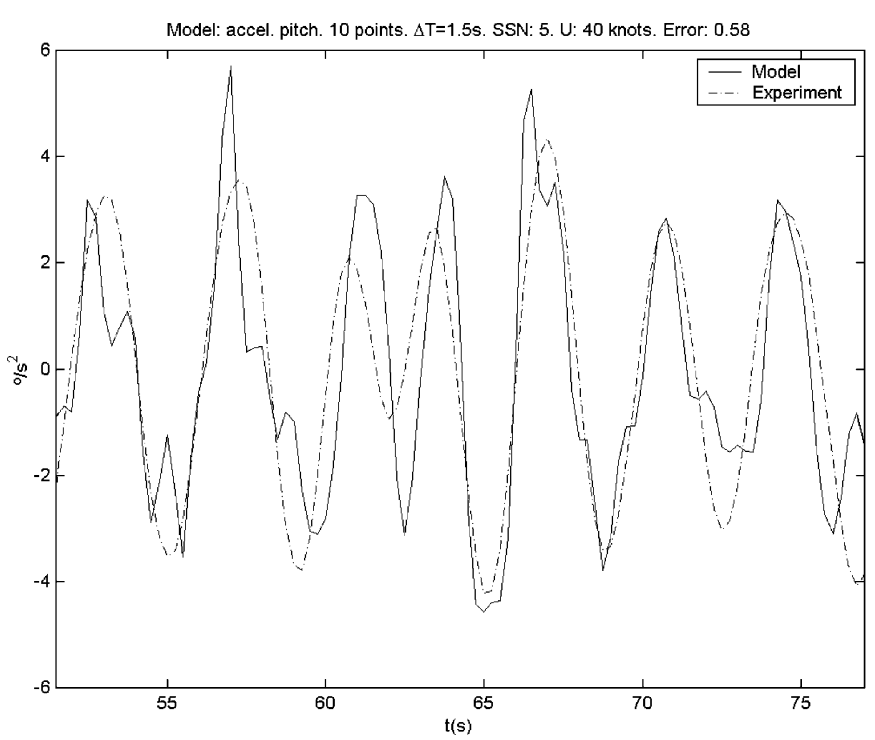

Fig. 16. Results for $\mathrm{SSN}=5 U=40$ knots 


\section{Conclusions}

Marine systems are known to be rather nonlinear with significant poorly understood influences. So, finding a general model of the system is not a trivial task, and it allows to try different control alternatives for the crafts.

In this paper, a general neuro-fuzzy model has been obtained for a fast ferry vertical motion. Based on physical principles, experimental and simulated data, and on the qualitative knowledge of its behaviour, a neuro-fuzzy inference system has been applied to estimate the nonlinear model. The model has been developed for pitch and heave motions and heading seas, using as inputs the sea state or modal frequency of the waves, the ship speed and the heading angle.

The neuro-fuzzy model shows good pattern with experimental and simulated data for regular waves, for different sea states, and has been compared with other models with satisfactory results. The results are original as other approximations to the problem do not cope with any sailing situation, whereas the neuro-fuzzy model is a general one in the sense that can be apply for any condition.

On the other hand, a predictive model has been obtained for irregular waves.

These control-oriented models have been developed in order to apply some neuro-fuzzy control strategies (Esteban et al., 2000; López and Santos, 2002; Santos et al., 2005). They have been used to improve the quality of the travelling by stabilising the motion of these high-speed vessels, as it is nowadays strongly demanded for different purposes.

\section{Acknowledgements}

Authors would like to thank the support of the CICYT TAP97-0607-C03-01 and DPI 2000-0386-C03-01 and the collaboration of the CEHIPAR staff.

\section{References}

Andres, B.D., Esteban, S., Giron, J.M., de la Cruz, J.M., 2000. Modelling the motions of a fast ferry with the help of genetic algorithms. In: Proceedings of the Third IMACS MATHMOD.

Anonymous, 1996. $126 \mathrm{~m}$ Long Spanish Fast Ferry Launched. Fast Ferries, September 19-20.

Anonymous, 1998. Silvia Ana: Results of First Year's Service. Ship \& Boat International, January 15-16.

Aranda, J., de la Cruz, J.M., Díaz, J.M., de Andrés, B. Ruipérez, P., Girón, J.M., 2000. Modelling of a high speed craft by a non-linear least squares method with constraints. In: Proceedings of the Fifth IFAC Conference on Manoeuvring and Control of Marine Crafts.

Aranda, J., Muñoz, R., Díaz, J.M., 2002. Comparison of the vertical dynamics models of a fast ferry with actuators and without actuators. In: Proceedings of the Third International Congress on Maritime Technological Innovations and Research, Bilbao, Spain.

Babuska, R., Verbruggen, H.B., 1996. An overview of fuzzy modelling and control. Control Engineering Practice 4, 1593-1606.

Brown, M., Harris, C., 1994. Neurofuzzy Adaptive Modelling and Control. Prentice-Hall International, Englewood Cliffs, NJ.
CEHIPAR, 1998. Inf. OTI-2135-CM-2 "Resultados del programa de comportamiento en la mar Precal del Ferry TF-120 para investigación de control". Madrid.

Chiu, S., 1994. Fuzzy model identification based on cluster estimation. Journal of Intelligent and Fuzzy Systems 2 (3).

de la Cruz, J.M., Aranda, J., Díaz, J.M., Ruipérez, P., Marón, A., 1998. Identification of the vertical plane motion model of a high speed craft by model testing in irregular waves. In: Proceedings of IFAC Conference Control Aplications in Marine Systems, pp. 277-282.

Esteban, S., Girón-Sierra, J.M., de la Cruz, J.M., de Andrés, B., Díaz, J.M., Aranda, J., 2000. Fast Ferry Vertical Acceleration Reduction with Active Flaps and T-Foil. In: Proceedings of the Fifth IFAC Conference on Manoeuvring and Control of Marine Crafts, pp. 233-238.

Fossen, T.I., 1994. Guidance and Control of Ocean Vehicles. Wiley, New York.

Harris, C.J., Moore, C.G., Brown, M., 1993. Intelligent Control-Aspects of Fuzzy Logic and Neural Nets. World Scientific, Singapore.

Jang, J.S.R., 1993. Anfis: adaptive-network-based fuzzy inference systems. IEEE Transactions on Systems, Man, and Cybernetic 23, 665-685.

Lewis, E.V., 1989. Principles of Naval Architecture. SNAME, New Jersey.

Lloyd, A.R.J.M., 1998. In: Horwood, E. (Ed.), Seakeeping: Ship Behaviour in Rough Water. RPM, Sussex, UK.

López, R., Santos, M., 2002. Neuro-Fuzzy system to control the fast ferry vertical acceleration. 15th IFAC World Congress, Barcelona.

López, R., Santos M., Polo, O., Esteban, S., 2002. Experimenting a fuzzy controller on a fast ferry. IEEE Conference on Control Applications/ Computer Aided Control Systems Design Conference.

O'Hanlon, J.F., McCawley, M.E., 1974. Motion sickness incidence as a function of acceleration of vertical sinusoidal motion. Aerospace Medicine 45 (4), 366-369.

Santos, M., López, R., de la Cruz, J.M., 2005. Fuzzy Control of the vertical acceleration of fast ferries. Control Engineering Practice 13/3, 305-313.

Ziegler, H., 1968. Principles of Structural Stability. Blaisdell, New York.

M. Santos received the M.S. degree in Physics (Computer Engineering) in 1986 and the Ph.D. degree in 1994 from the University Complutense of Madrid (UCM). Since 1994 she has been with the Department of Computer Architecture and Automatic Control, where she is engaged in teaching and research in the area of intelligent control, modelling, simulation, and signal processing. Since 1997 she has been a senior Reader of System Engineering in Computer Science. She is the author and coauthor of several journal and conference papers in the related fields. Her current research interests include fuzzy (adaptive neuro-fuzzy control and modelling), discrete events simulation, pattern recognition (clustering), wavelets.

R. López received the M.E. degree in Electrical and Computer Engineering from the University Complutense of Madrid (UCM), and the Ph.D. degree in System Engineering at Complutense University (2004). His current research interests include control system, neural networks, fuzzy logic theory and genetic algorithms.

J.M. de la Cruz received the M.E. and Ph.D. degrees in Physics from University Complutense of Madrid, in 1979 and 1984, respectively. Since 1990 he has been a Professor of System Engineering in the Department of Computer Engineering. He has authored and co-authored more than 200 journals and contributed papers at all major conferences in the related field. His current research interests include optimisation, control theory, real time control, artificial vision, etc. $\mathrm{He}$ has been the Principal Investigator in EU and industrially funded projects. His research group has an international acknowledgment. 